\title{
Interstitial Boron in Tungsten: Electronic Structure, Ordering Tendencies, and Total Energy Calculations
}

\author{
VLAD LIUBICH, ${ }^{1}$ DAVID FUKS ${ }^{1} *{ }^{1}$ SIMON DORFMAN ${ }^{2}$ \\ ${ }^{a}$ Department of Materials Engineering, Ben-Gurion University of the Negev, POB 653, Beer-Sheva, \\ Israel \\ ${ }^{2}$ Faculty of Physics, Israel Institute of Technology-Technion, 32000 Haifa, Israel
}

Received 12 May 1999; accepted 13 May 1999

\begin{abstract}
Small amounts of additives sufficiently change the structure of the parent lattice. Boron forms dilute interstitial solid solutions in the tungsten host. In the framework of liner density functional theory, we study the conditions of the formation of $\mathrm{W}-\mathrm{B}$ solid solutions. On the basis of the coherent potential approximation (CPA), we consider ordering tendencies, study the electronic structure, and provide total energy calculations. Results of nonempirical calculations predict the anomalous behavior of the coefficient of concentration dilatation of the lattice and a nonmonotonic behavior of electron density of states at the Fermi energy with the concentration dilution. (c) 1999 John Wiley \& Sons, Inc. Int J Quant Chem 75: 917-926, 1999
\end{abstract}

Key words: electronic structure; solid solutions; tungsten; $\mathrm{W}-\mathrm{B}$; $\mathrm{CPA}$

\section{Introduction}

$\mathrm{n}$ the case of many metals and alloys, small boron additions modify their ambient tempera-

Correspondence to: V. Liubich.

*On sabbatical stay in The Institute of Physics, Federal University of Bahia, Salvador, Brazil.

Contract grant sponsor: Israel Science Foundation.

Contract grant number: 380/97-11.7. ture properties (see, e.g., [1]). In both $\mathrm{Fe}$ and $\mathrm{W}$, impurities such as $\mathrm{N}, \mathrm{O}, \mathrm{P}, \mathrm{S}$, and $\mathrm{Si}$ weaken the intergranular cohesion, resulting in "loosening" of the grain boundary (GB). The presence of B and C, on the contrary, enhances the interatomic interaction across the GB. Boron plays a dual role in both Fe and $\mathrm{W}$; not only does its presence at GBs enhance the integranular cohesion, but it also accomplishes "site competition cleaning" (SCC) by displacing the other impurity atoms off the GB. Microalloying with 10-50 ppm B may be an effec- 
tive way of improving the ductibility of both Febase alloys and W [2]. Some intermetallic alloys, which present an intrinsic intergranular brittleness in their undoped state, change their fracture mode when boron-doped. In some cases-as in the Bdoped hypostoichiometric $\mathrm{Ni}_{3} \mathrm{Al}$ alloys - the fracture becomes ductile. In other cases, as in FeAl (B2) alloys, even in the B-doped alloys, a brittle fracture is observed, but it takes place in a transgranular manner by cleavage [3, 4].

The boron effects in metals and alloys are typically attributed to its intergranular segregation. This hypothesis is a conclusion of experimental measurements of some integranular boron enrichment, mainly in $\mathrm{Ni}_{3} \mathrm{Al}$ alloys, by Auger electron spectroscopy (AES) [5]. For the case of tungstenbased solid solutions, the nonempirical calculations were used to study the influence of alloying on the ductile-brittle phase transformation $[2,6]$. On the basis of these simulations, a statement was formulated that the cohesion of a GB is believed to be the controlling factor limiting the ductility of high-strength metallic alloys, and particularly those containing W. Intergranular embrittlement is usually associated with segregation of impurities at the GBs. SCC should play an important role affecting impurity distribution in W GBs. Among the impurities analyzed, B in the GB has the lowest energy and thus would tend to displace other impurity atoms from the GB. Microalloying with 10-50 ppm B may be an effective way of improving tungsten's ductility. These results are important for understanding the fundamental physics of intergranular embrittlement. As a result, a number of articles were devoted to the modeling of tungsten GBs in different approaches (see, e.g., [7] and references therein). However, as far as we know, no systematic study of the tungsten-based solid solutions in its thermodynamic aspects is available in the existence literature.

The purpose of our work was an extensive study of tungsten-based solid solutions, with the emphasis put on the modeling of the electronic structure, ordering tendencies, and total energy studies. The importance of the boron presence for the bulk properties of the interstitial solid solution is also shown.

\section{LDA Model}

The essential requisite for such research is to have a reliable and efficient electronic-structure method for the calculation of the total energies and electronic structures of the interstitial solid solutions of tungsten. Within the framework of the Hohenberg-Kohn-Sham density functional theory (DFT), the electronic structure can be efficiently handled using a first-principles self-consistent theory. DFT has wide applications to molecules and solids [8]. Even the crudest approximation, the local density approximation (LDA), to the density functional theory has been successfully applied to predict structural and dynamical properties of a large variety of materials. Equilibrium volumes, elastic constants, phonon frequencies, surface reconstruction, and magnetism are just some examples of properties which could be successfully calculated for systems without particularly strong electron correlations within the LDA. The LDA usually leads to some overbidding in solids (equilibrium volumes are typically $1-3 \%$ underestimated). Considerably larger errors are found in cases where the LDA is not sufficiently accurate; the ionic compounds like $\mathrm{MgO}$ serve as examples when the simple LDA fails.

The electronic structure of crystalline solids could be efficiently calculated using the linear band-structure methods. The linear muffin-tin orbitals (LMTO) method is particularly fast and efficient for handling complex and large unit cells because of the ease with which the structure-dependent part and the potential-dependent part are separated out in the secular equation [9]. An important milestone in the application of the LMTO was understanding that the original infinite-ranged LMTO basis set can be limited only by few orbital functions. With this advantage, the LMTO method has the computational simplicity of the empirical tight-binding schemes, as well as on accuracy characteristic for a first-principles method.

LMTO in the atomic sphere approximation (ASA) approach is a well-established technique to study metals, alloys, and also perovskites and different phases with nontrivial and sometimes very complicated structures [10-13]. For close-packed metallic structures, in particular, this method consistently predicts reliable and accurate results comparable to those obtained from other sophisticated LDA-based methods like the linearized augmented plane wave (LAPW) [14]. The systematic errors due to the use of ASA cancel out while determining the ground-state energy differences, such as formation energies. In the last years, it was successfully applied to the study of surface alloys 
(see, e.g., [13]. In the case of dilute alloys, the properties of Fe embedded in $\mathrm{V}$ and $\mathrm{Cr}$ matrices was successfully studied [15]. It seems that today this scheme is one of the most promising techniques in band-structure studies due to the ability to calculate very fast very complicated structures. The LMTO ASA method is ideally suited to the relatively close-packed solid solutions of the tungsten treated here, where one can ensure a reasonably small overlap between the atomic spheres. We reduced the value of this overlap by introducing an additional interstitial ("empty") sphere. In the LMTO ASA, the approximation due to spherical averaging is also controlled by changing the ratio between atomic spheres of different species. The overlap between the spheres is defined as $\left(S_{1}+S_{2}-d\right) \times 100 / S_{1}$ and is less than $30 \%$. Here, $S_{1}$ and $S_{2}\left(S_{1}<S_{2}\right)$ are the radii of the two overlapping spheres and $d$ is the distance between them. By incorporating the so-called combined correction, one can partly reduce the error due to a spherical form of potential and charge density [9].

To study the effects of the electronic-density distributions in tungsten-based solid solutions on chemical bonding, we modeled different tungsten-based cells. The behavior of additives in tungsten were studied on the basis of the firstprinciples total-energy calculations of binary, partially ordered compounds in the coherent potential approximation (CPA). The total energies were calculated for varying lattice constants [i.e., WignerSeitz (WS) radii] to determine the relative stability of different structures and to locate the equilibrium lattice constant. From the total energy of the phase and constituent elemental solids, one can find the formation energy using the expression

$$
\begin{aligned}
E_{\text {form }}^{W-\text { dopant }}=E_{\text {total }}^{W-\text { dopant }} & \\
& -\left[c E_{\text {total }}^{W}+(1-c) E_{\text {total }}^{\text {dopant }}\right] .
\end{aligned}
$$

Here, $c$ is the concentration of tungsten, $E_{\text {total }}^{W-\text { dopant }}$ refers to the total energy per formula unit of the crystalline phase at equilibrium lattice constants, and $E_{\text {total }}^{W}$ and $E_{\text {total }}^{\text {dopant }}$ represent the total energies of tungsten and the dopant, respectively. Following this line, the systematic errors in total energy caused by ASA can be eliminated effectively.

A decomposition of the total energy of a random alloy into partial contributions

$$
E_{\text {rand }}^{W-\text { dopant }}(c)=c E_{W}(c)+(1-c) E_{\text {dopant }}(c)
$$

is not, in general, possible. $E_{W}(c)$ and $E_{\text {dopant }}(c)$ are the partial contribution to the total energy from the alloy components. However, it exists in ASA, where the total energy of an alloy with an arbitrary configuration may be written for $N$ atoms in the form

$$
E_{\text {tot }}=\sum_{i=1}^{N} \varepsilon_{i}
$$

where $N$ is the number of atoms in the system and $\varepsilon_{i}$ is the local contribution to the

$$
\begin{aligned}
E_{W}(c) & =\frac{1}{N_{W}} \sum_{i}\left\langle\varepsilon_{i}(c) c_{i}\right\rangle \quad \text { and } \\
E_{\text {dopant }}(c) & =\frac{1}{N_{\text {dopant }}} \sum_{i}\left\langle\varepsilon_{i}(c)\left(1-c_{i}\right)\right\rangle
\end{aligned}
$$

total energy from an atom on the site $i$. Hence, we may use ASA in calculations of total energies as long as the geometry of the lattice is fixed. In this case, the on-site energies are determined as the average quantities [16] and the total energy of a random alloy may be rewritten

$$
E_{\text {rand }}(c)=\frac{1}{N} \sum_{i}\left\langle\varepsilon_{i}(c)\right\rangle .
$$

Here, $N_{W}$ and $N_{\text {dopant }}$ are the number of tungsten and dopant atoms. $N=N_{W}+N_{\text {dopant }}, c=N_{W} / N$, $c_{i}$ is an occupation number taking on the values 1 and 0 depending on whether site $i$ is occupied by tungsten or a dopant, respectively. The angle brackets, \langle\rangle , mean averaging over configurations.

Band-structure calculations based on the DFT allow one to obtain a quantitative description of the ground-state properties of absolutely ordered alloys. Application of these methods to the calculations of the thermodynamic properties of partially ordered or random alloys gives reasonable results $[17,18]$. The most attractive feature of the singlesite CPA is the ability to apply this scheme to the direct calculations of the electronic structure of randomly or partially ordered alloys [18]. Recent applications of the CPA scheme show that this method allows one to reproduce accurately the lattice parameters, bulk moduli, and enthalpies of formation (see, e.g., [19] and references therein). This accuracy is similar to the accuracy of other local density functional methods for completely ordered phases. 


\section{CPA Formalism}

An approach based on direct calculations of the electronic structure of random or partially ordered alloys is the single-site (SS) CPA, which gives very similar values for the equilibrium lattice parameter, bulk moduli, and enthalpies of formation to that obtained by ordinary first-principles methods. We present herewith only a brief introduction into the technique of the usual SS CPA for solids with several different sublattices [19].

Let us consider an ordering $W_{c} B_{1-c}$ alloy with $k$ $\alpha$-type sublattices and $m \beta$-type sublattices, where $c$ is the concentration of the component $W$. Thus, in the completely ordered state, the alloy has the formula $W_{k} B_{m}$. Next, we can suggest, for simplicity, that all the $\alpha$ as well as all the $\beta$ sublattices are equivalent. To describe a partially ordered state of the alloy, it is important to represent a single long-range order (LRO) parameter $\eta$. It is defined by

$$
\eta=c_{\alpha}-c_{\beta}
$$

where $c_{\alpha}$ and $c_{\beta}$ are concentrations of the component $A$ on the $\alpha$ and $\beta$ sublattices, respectively. $c_{\alpha}$ and $c_{\beta}$ can be written as

$$
c_{\alpha}=c+\frac{m}{n} \eta \quad \text { and } \quad c_{\beta}=\frac{k}{n} \eta,
$$

where $n=k+m$ is the total number of sublattices in the alloy. Each sublattice is considered a completely random alloy with corresponding concentrations of $W$ and $B$ components. Thus, in the SS approximation, the partially ordered alloy is described as a set of coexisting completely random alloys.

The average one-electron Green's function should be determined for calculations of electronic structure and ground-state properties. To obtain this function, we apply the SS CPA in conjunction with the LMTO method in the ASA. The average one-electron Green's function may be obtained in the form of the Korringa-Kohn-Rostoker (KKR) ASA Green's function, which is identical to the scattering path operator in multiple scattering theory. For a complex energy $z$, we have

$$
\Gamma_{i j}(z)=\frac{1}{V_{B Z}} \int_{B Z} d^{3} k[\Re(z)-\Lambda(\mathbf{k})]_{i j}^{-1},
$$

where $V_{B Z}$ is the volume of Brillouin zone (BZ), $\Lambda(\mathbf{k})$ are the LMTO structure constants, and $\Re(z)$ is the crystal coherent-potential matrix. The subscripts $i$ and $j$ refer to individual sublattice sites in the unit cell. We have omitted the angular momentum quantum numbers $\left(l_{m}\right)$ as well as the LMTO representation number. The coherent-potential matrix that enters Eq. (3) is block-diagonal:

$$
\Re=\left(\begin{array}{cccc}
\mathfrak{R}_{1} & 0 & \cdots & 0 \\
0 & \Re_{2} & \cdots & 0 \\
\cdots & \cdots & \cdots & \ldots \\
0 & 0 & \cdots & \mathfrak{R}_{n}
\end{array}\right)
$$

and each diagonal element $\Re_{i}$ is the coherentpotential function of the $i$ sublattice. To obtain the complete coherent-potential function, we must solve for each sublattice the following equation:

$$
\begin{aligned}
\Re_{i}=c_{i} \Re_{i}^{W}+(1 & \left.-c_{i}\right) \Re_{i}^{B} \\
& +\left[\Re_{i}^{W}-\Re_{i}\right] \Gamma_{i i}\left[\Re_{i}^{W}-\Re_{i}\right],
\end{aligned}
$$

where $c_{i}$ is the concentration of component $A$ and $\mathfrak{R}_{i}^{k}$ is the potential function of the $k$ species on sublattice $i$. In the $\alpha$ representation of the LMTO method, the potential function may be found in the form

$$
\mathfrak{R}_{i l}^{\alpha \kappa}(z)=\frac{C_{i l}^{\kappa}-z}{\left(C_{i l}^{\kappa}-z\right)\left(\gamma_{i l}^{\kappa}-\alpha_{l}\right)+\Delta_{l l}^{\kappa}}
$$

in terms of the LMTO parameters [9] $C_{i l}^{k}, \Delta_{i l}^{k}$ and $\gamma_{i l}^{k}$ obtained from the solution of the radial Schrödinger equation at some fixed energy $E_{\nu}$ with an angular momentum $l$. Coherent-potential functions are coupled by the definition [Eq. (3)] of the coherent Green's function, which, together with Eq. (5), form the nonlinear system of CPA equations that must be solved self-consistently.

We calculated the electronic-density distributions with the CPA LMTO code $[15,19]$. This code includes the determination of the Madelungenergy prefactor $\zeta$, which makes the CPA LMTO results agree with those obtained by the Connolly-Williams method on the basis of the total energies of ordered alloys [17]. This prefactor enters into the expression for the Madelung energy of the alloy, and for the case of the tungsten-based solid solution it is

$$
E_{\text {Mad }}=-\zeta e^{2} c(1-c) \frac{\left(Q_{W}-Q_{\text {dopant }}\right)^{2}}{R(1)} .
$$


Here, $R(1)$ is the radius of the first coordination shell, $e$ is the electron charge, and the tungsten and dopant charges are $Q_{W}$ and $Q_{\text {dopant }}$, respectively. These charges are defined as

$$
\begin{aligned}
Q_{W} & =\int_{S_{W S}} d^{3} r \rho_{w}-Z_{W}, \quad \text { and } \\
Q_{\text {dopant }} & =\int_{S_{\text {w s }}} d^{3} r \rho_{\text {dopant }}-Z_{\text {dopant }} .
\end{aligned}
$$

In these equations, $S_{w s}$ is the radius of atomic sphere, $Z_{W}$ and $Z_{\text {dopant }}$ are the atomic numbers of tungsten and the dopant, and $\rho_{w}$ and $\rho_{\text {dopant }}$ are the electronic densities of tungsten and the dopant, respectively. A number of models could be reduced to Eq. (7). The only difference will be the value of prefactor $\zeta$. In [20], it was demonstrated that a single value of the prefactor can be chosen for all concentrations and values of the lattice parameters; it varies from system to system. Advances of our CPA calculations are in the use of the total and not one-electron energies, in the inclusion of charge-transfer effects in CPA, and in our refusal to use the effective pair interactions.

\section{Calculations}

Our study was based on the analysis of electronic-density distributions for diffeent interatomic distances, supercell configurations, and compositions of an interstitial impurity. Changes in the concentration will lead to changes of the supercell volume and to changes in the character of the bonding forces. Band structures of a completely ordered tungsten-based interstitial alloy with partially occupied octahedral positions

$$
\left(\begin{array}{lll}
\frac{1}{2} & \frac{1}{2} & 0
\end{array}\right) \quad \text { and } \quad\left(\begin{array}{lll}
\frac{1}{2} & 0 & 0
\end{array}\right)
$$

were carried out within the CPA LMTO procedure briefly outlined in the previous section [19]. The studied structures are presented in Figure 1. With the self-consistently obtained bands, we calculated the equilibrium total energies of the completely ordered phases and phases with partially occupied sublattices. The LMTO scheme permits a localized expansion of electronic wave functions, densities, and derived properties by treating fragments of the extended system in the supercell approach [9]. This approach gives a feasible methodology for

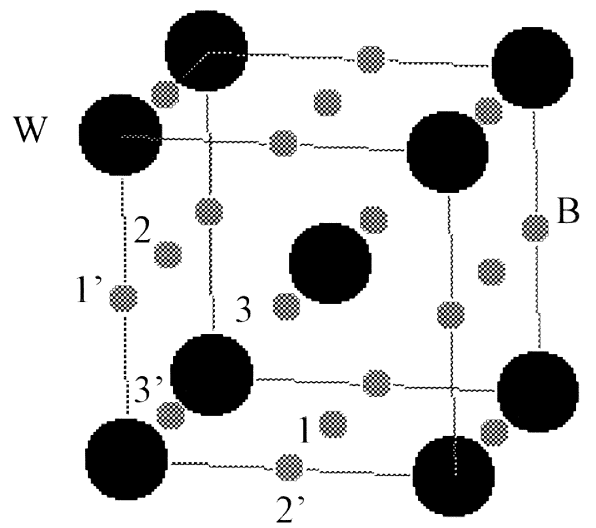

FIGURE 1. Structure of tungsten-boron supercell. Nos. 1, 2, and 3 define the sublattices of octahedral interstitial positions.

self-consistently treating large systems with low symmetry and is, thus, particularly well suited for problems of impurities, surfaces, and interfaces $[19,20]$.

\section{Results and Discussion}

In our calculations, we studied the behavior of the dilute solid solutions of boron in tungsten. According to the Hagg's rule, we assumed that these solutions are interstitial, because the ratio of atomic radii of constituents is less than 0.49 . Boron atoms were distributed on one of three sublattices of the octahedral interstitial positions of the host matrix. As may be seen from Tables I-III, the atomic fraction of boron did not exceed 0.167 . Such a modeling had two main aims: (a) to study the tendencies of the ordering of boron atoms on one of the interstitial site sublattices in tungsten and (b) to obtain knowledge about the bonding tendencies for boron atoms in tungsten media.

\section{TABLE I}

Partial total energies per cubic cell for tungsten and boron atoms.

\begin{tabular}{lll}
$\begin{array}{l}\text { Atomic } \\
\text { fraction of } B\end{array}$ & $E_{W}$ (in Ry) & $E_{B}$ (in Ry) \\
\hline 0.001 & -16.123054 & -6.137887 \\
0.01 & -16.130929 & -6.118304 \\
0.1 & -16.172706 & -5.878522 \\
0.167 & -16.244500 & -5.742988
\end{tabular}


TABLE II

Total number of states $\left(T_{\mathrm{NOS}}\right)$ for tungsten and boron atoms at the Fermi energy, $E_{F}$, and total energy, $E_{\text {tot }}$, per atom for W-B dilute solid solutions.

\begin{tabular}{llccc}
\hline $\begin{array}{l}\text { Atomic } \\
\text { fraction of B }\end{array}$ & $T_{\text {NOS }}(\mathrm{W})$ & $T_{\text {NOS }}(\mathrm{B})$ & $\begin{array}{c}\text { Fermi energy, } \\
E_{F} \text { (in Ry) }\end{array}$ & $\begin{array}{c}\text { Total energy, } \\
E_{\text {tot }} \text { (in Ry) }\end{array}$ \\
\hline 0.001 & 4.959377 & 2.554522 & 0.273295 & -8.056310 \\
0.01 & 4.974857 & 2.550489 & 0.271708 & -8.079654 \\
0.1 & 5.185947 & 2.518177 & 0.196862 & -8.345946 \\
0.167 & 5.349266 & 2.480321 & 0.185083 & -8.580559 \\
\hline
\end{tabular}

TABLE III

Partial $T_{\text {NOS }}$ for $W$ and for different compositions of solid solutions.

\begin{tabular}{|c|c|c|c|c|c|c|}
\hline \multirow{2}{*}{$\begin{array}{l}\text { Atomic } \\
\text { fraction of } B\end{array}$} & \multicolumn{3}{|c|}{ W } & \multicolumn{3}{|c|}{ B } \\
\hline & $s$ & $p$ & $d$ & $s$ & $p$ & $d$ \\
\hline 0.001 & 0.624397 & 0.496280 & 3.838700 & 0.665179 & 1.653194 & 0.236149 \\
\hline 0.01 & 0.625342 & 0.506733 & 3.842782 & 0.664970 & 1.649617 & 0.235902 \\
\hline 0.1 & 0.649117 & 0.632778 & 3.904052 & 0.676672 & 1.612493 & 0.229011 \\
\hline 0.167 & 0.656756 & 0.728383 & 3.964127 & 0.674390 & 1.576550 & 0.229382 \\
\hline
\end{tabular}

As the first step of the calculations, we investigated the structure where boron occupies in small concentrations only the corners of one cubic primitive cell of the octahedral interstitial positions. At each stage of the calculations, that is, at all the studied concentrations, the lattice parameter was varied to obtain the minimal total energy. As seen from Table I, the increase of boron concentration has an opposite effect on the partial total energies: The tungsten energy decreases while the boron energy increases. The same opposite tendency is illustrated by Table II: The total number of states at the Fermi energy for $\mathrm{V}$ increases while for $\mathrm{B}$ it decreases with the growth of the boron concentration. At the same time, the total energy per atom for dilute $W-B$ interstitial solid solutions decreases as well as the Fermi energy.

In Table III, we present the results of the calculations of the partial total numbers of states $\left(T_{\text {NOS }}\right)$ for $\mathrm{W}$ and $\mathrm{B}$ atoms for different compositions of solid solutions. The partial $s-$ and $p-T_{\text {NOS }}$ for $\mathrm{W}$ show the stable tendency to increase with the rising boron concentration while $s$ - and $p-T_{\text {NOS }}$ for $B$ decrease. This is not case for the behavior of $d-T_{\text {NOS }}$. For $W$, it increases, while for boron atoms when the concentration is decreasing and at a concentration $c=0.167$, it starts to increase, showing, thus, a nonmonotonic behavior. In Figure 2, we present the dependence of the energy per atom on the lattice parameter for two concentrations of extremely dilute solid solutions. The presented curves show nontrivial behavior. We may analyze these curves in terms of the enthalpy because the pressure-volume term in the units, which are used for calculations, is negligibly small. By drawing a common tangent to the curve in points $A$ and $B$ in this figure, we find that, for example, for the lattice parameter of $6.2 \mathrm{au}$ and the atomic fraction of boron equal to 0.001 , the two-phase mixture of dilute solid solutions with lattice parameters 6.14 and $6.24 \mathrm{au}$ is preferable in comparison with the one-phase state of the solid solution. This means that, although for pressure equal to zero, the onephase state with the lattice parameter $a=6.1$ au is stable; when tensile stress is applied, the solid solution decomposes into a two-phase state. When the concentration of boron increases, the effect weakens as shown by the dashed line in Figure 2 and vanishes at a larger concentration of boron.

Additional information may be obtained from the study of the concentration dependence of the total energy of extremely dilute $\mathrm{W}-\mathrm{B}$ solutions. Figure 3 and 4 present this dependency. From Figure 3, it follows that both the energy and lattice parameters are nonlinear functions of the concentration. It may be seen in Figure 3 that at an extremely small boron concentration the lattice parameter has an anomalous behavior. Figure 4 presents a "microscope" study of this part of Figure 3. It is easy to see that the coefficient of concentration 


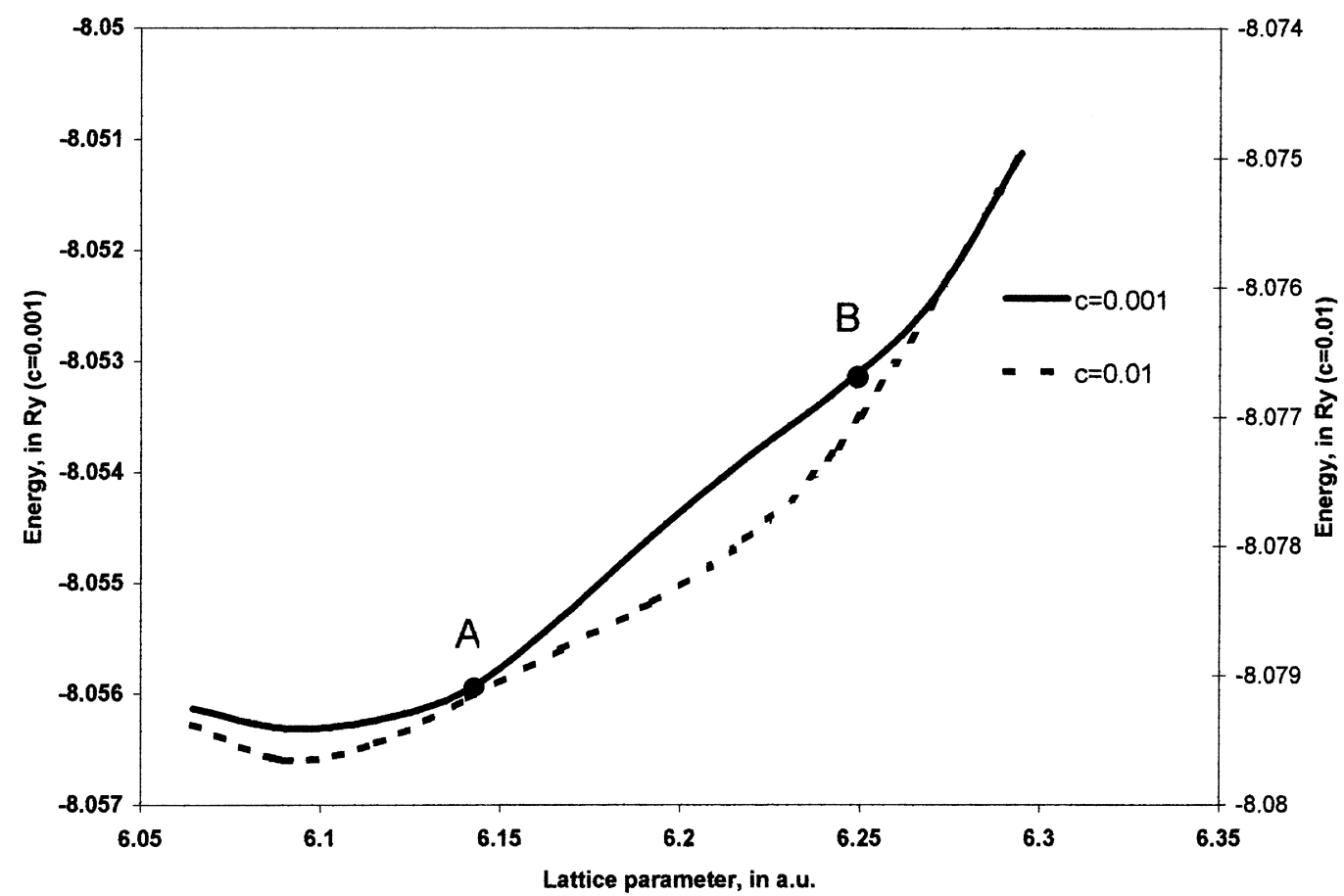

FIGURE 2. Dependence of the energy per atom on the lattice parameter for different atomic fractions of boron.

dilatation of the lattice, $u=a_{0}^{-1}(d a / d c)$, is negative for the atomic fraction of $\mathrm{B}$ until 0.006 , becomes equal to zero for this composition, and, after this, becomes positive and concentrationindependent.

As the second step of our calculations, we studied the different possibilities of occupations of the sites of one sublattice of the octahedral positions of boron atoms. The results of this study are given in Tables IV and V. In Table IV, we show how the occupation influences the equilibrium WignerSeitz radius and the total energy of the solid solution. The presented results clearly demonstrate the tendency to form the $\mathrm{B} 2$ phase ( $\mathrm{CsCl}$ type) on the sublattice of octahedral interstitial positions. This B2 phase consists of boron atoms which occupy the corners of the cubic cell of the sublattice while the centers of this cell are empty. This configuration is favorable in comparison with others studied if the energies are related. In Table V, we illustrate the influence of the changes of boron concentration on the energy of extremely dilute interstitial solid solutions. In these calculations, boron atoms were homogeneously distributed on the sublattice of octahedral interstitial positions. In Figure 5, the total densities of states for dilute $\mathrm{W}-\mathrm{B}$ alloys are presented for different compositions. The displayed plots clearly demonstrate the formation of an additional band in the low-energy region when the boron concentration increases. This fact indicates the formation of chemical bonding in the $\mathrm{W}$ -B solid solution. Our study of partial DOSs of tungsten and boron shows that this bonding is provided mainly by the interaction of low-lying $s$ and $d$ bands of tungsten with $s$ bands of boron. The DOS at the Fermi level, $N\left(E_{F}\right)$, is an important quantity as it is used for the estimation of the electronic specific heat and the electron-photon coupling constant and even for determining the vibration contribution to the entropy at finite temperature. The numbers of $N\left(E_{F}\right)$ reflect the trend of metallicity in this solid solution. Figure 6 displays the behavior of the total density of states in the vicinity of the Fermi energy. It is seen that increase of the atomic fraction of boron leads to decrease of the number of states at the Fermi level, thus decreasing the degree of the metallicity in the system. At the same time for $c_{B}=0.167$, we find, once more, the increase of $N\left(E_{F}\right)$. This conclusion is confirmed by the results given in Table VI, which shows the nonmonotonic change of $N\left(E_{F}\right)$ as a function of the boron concentration. 


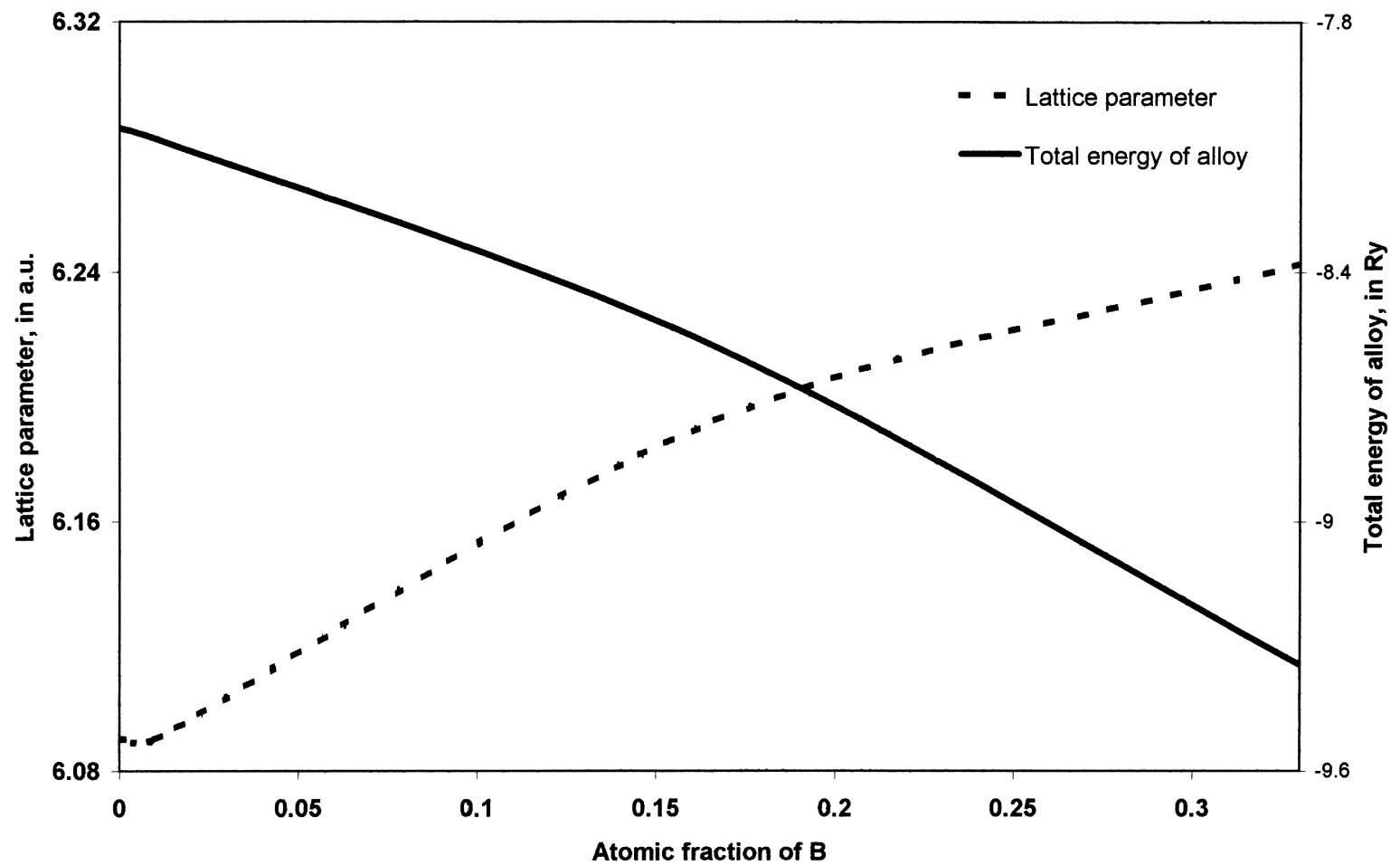

FIGURE 3. Dependence of the lattice parameter and the energy of tungsten-boron alloy on the atomic fraction of boron.

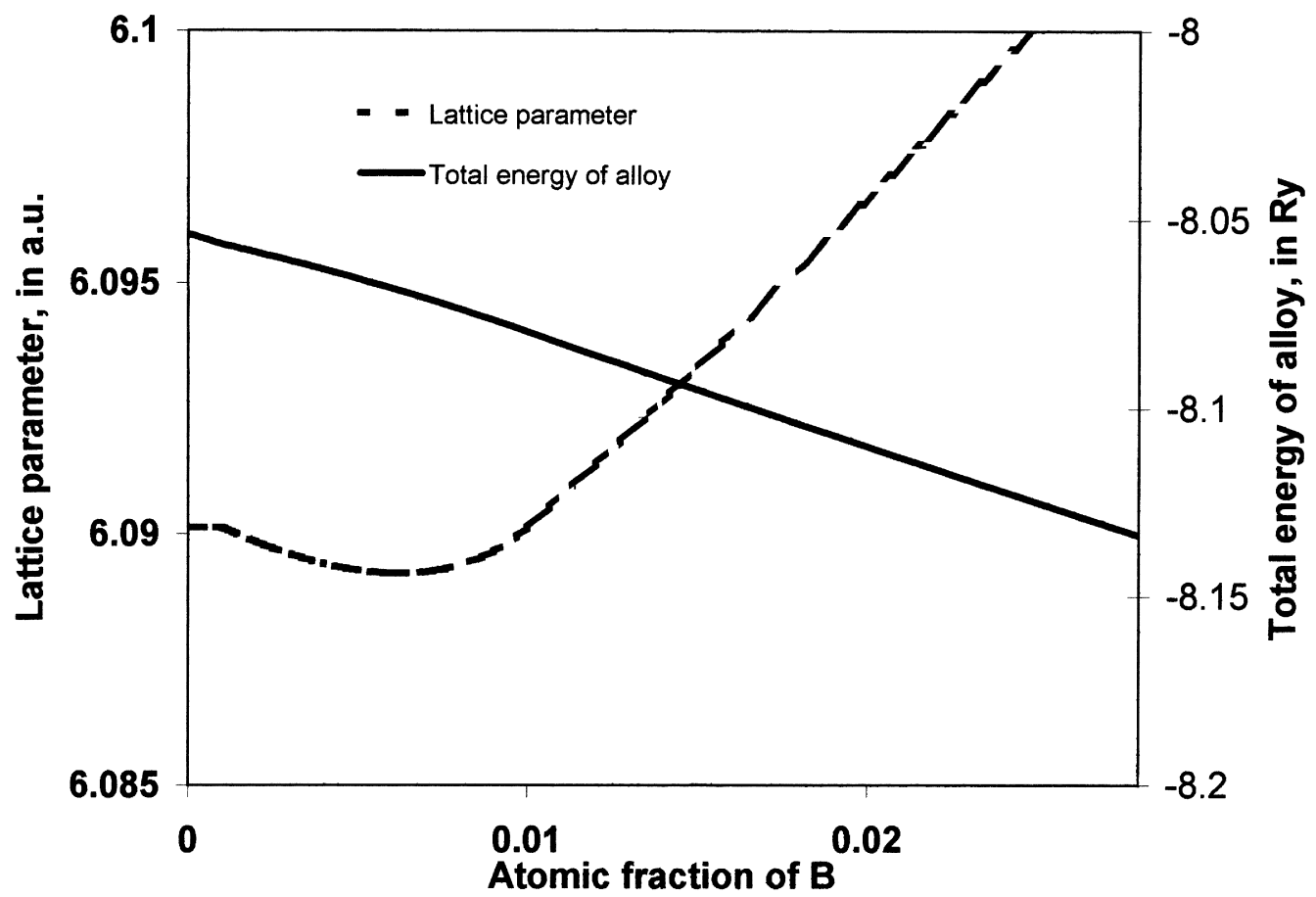

FIGURE 4. "Microscope" of Figure 3 for extremely dilute W-B solid solutions. 
TABLE IV

Total energies per atom for $\mathbf{W}$-B solid solution versus equilibrium Wigner-Seitz radius, $R_{w s}$ for different occupations of the sites in sublattice of octahedral interstitial positions.

\begin{tabular}{llll}
\hline & \multicolumn{2}{c}{ Occupation } & \\
\cline { 2 - 3 }$R_{\text {ws }}$ (in au) & $\mathrm{B}_{1}$ & $\mathrm{~B}_{1^{\prime}}$ & $E_{\text {tot }}$ (in Ry) \\
\hline 2.38 & 0 & 0 & -8.053733 \\
2.45 & 1 & 0 & -9.413844 \\
2.44 & 0.9 & 0.1 & -9.393629 \\
2.44 & 0.5 & 0.5 & -9.359033 \\
\hline
\end{tabular}

\section{Summary}

In this article, we studied the electronic structure and the total energy characteristics of an extremely dilute interstitial tungsten-boron solid solution. We discussed the tendencies of chemical bonding in such solutions and showed the possibility to form some type of ordering on the sublattice of interstitial octahedral positions. We pre-
TABLE V

Total energies per atom in W-B solid solutions for different concentrations of boron.

\begin{tabular}{|c|c|c|c|}
\hline \multirow{2}{*}{$\begin{array}{l}\text { Wigner -Seitz } \\
\text { radius, } \\
R_{w s} \text { (in au) }\end{array}$} & \multicolumn{2}{|c|}{ Occupation } & \multirow{2}{*}{$\begin{array}{c}\text { Total energy, } \\
E_{t o t} \\
\text { (in Ry) }\end{array}$} \\
\hline & $\mathrm{B}_{1}$ & $B_{1}$ & \\
\hline 2.38 & 0 & 0 & -8.053733 \\
\hline 2.38 & 0.001 & 0.001 & -8.056310 \\
\hline 2.38 & 0.01 & 0.01 & -8.079654 \\
\hline 2.38 & 0.2 & 0.2 & -8.579468 \\
\hline
\end{tabular}

sented the results of nonempirical calculations which predict the anomalous behavior of the coefficient of concentration dilatation of the lattice and formation of a two-phase mixture in these solid solutions when external tensile stress is applied.

\section{ACKNOWLEDGMENTS}

This work was supported by the Israel Science Foundation founded by the Israel Academy of Sciences and Humanities (Grant \#380/97-11.7). We are greatly indebted to Dr. A. Ruban for support during our CPA calculations.

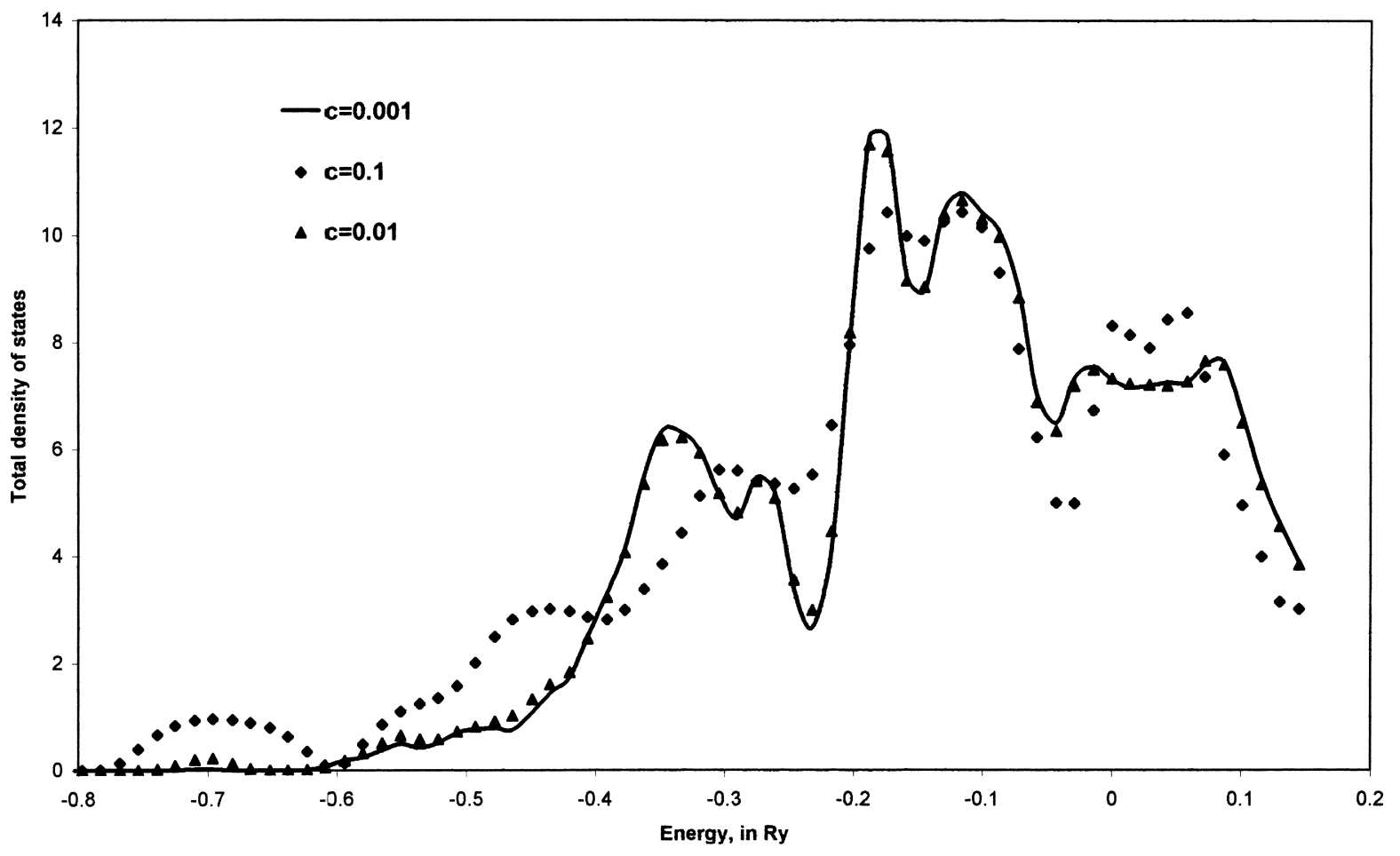

FIGURE 5. Total density of states in dilute interstitial W-B solid solutions for different atomic fractions of boron. 


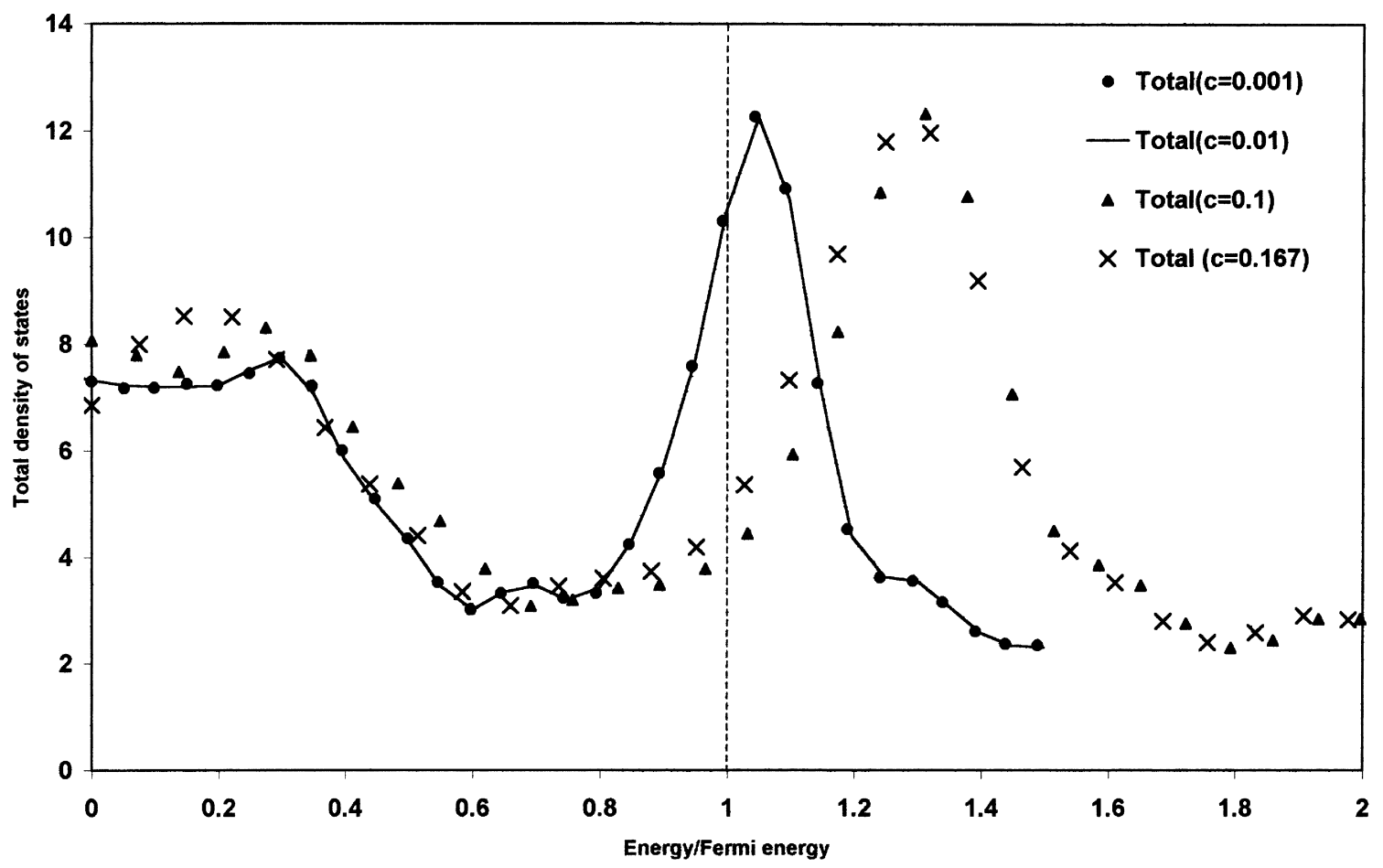

FIGURE 6. The same as Figure 5 in the vicinity of the Fermi energy.

\section{TABLE VI}

\section{Dependence of $N\left(E_{F}\right)$ on atomic fraction of boron.}

\begin{tabular}{lcr}
\hline $\begin{array}{l}\text { Atomic } \\
\text { fraction of B }\end{array}$ & $E_{F}$ (in Ry) & $N\left(E_{F}\right)$ \\
\hline 0.001 & 0.273 & 10.307 \\
0.01 & 0.271 & 10.476 \\
0.1 & 0.196 & 4.112 \\
0.167 & 0.185 & 4.945 \\
\hline
\end{tabular}

\section{References}

1. Baker, I.; Li, X.; Xiao, H.; Carleton, R.; Georege, E. P. Intermetallics 6, 177.

2. Krasko, G. L. Mat Sci Eng A 1997, 234-236, 1071.

3. Liu, C. T.; Lee, E. H.; McKamey, C. G. Scr Met 1989, 23, 875.

4. Franczkiewicz, A.; Gay, A.-S.; Biscondi, M. Mat Sci Eng A 1998, 258, 108.

5. Chuang, T. H. Mater Sci Eng A 1991, 141, 169.

6. Krasko, G. C. Int J Refract Hard Met 1993-1994, 12, 251.

7. Šob, M.; Wang, L. G.; Vitek, V. Mat Sci Eng A 1997, 234-236, 1075.
8. For example, see, "Electronic Density Functional Theory of Molecules, Clusters, and Solids," Ellis, D. E., Ed.; Kluwer, Dordrecht, 1995.

9. Andersen, O. K.; Phys Rev B 1975, 12, 3060; Skriver, H. L. The LMTO Method; Springer: New York, 1984.

10. Ellis, D. E.; Dorfman, S.; Fuks, D.; Evenhaim, R.; Mundim, K. C. Int J Quantum Chem 1998, 70, 1085.

11. Eriksson, O.; Johansson, B.; Brooks, M. S. S.; Skriver, H. L. Phys Rev B 1989, 40, 9519.

12. Antonov, V. N.; Yavorsky, B. Yu; Shpak, A. P.; Antonov, Vi. N.; Jepsen, O.; Guizzetti, G.; Marabelli, F. Phys Rev B 1996, 53, 15631.

13. Felser, C.; Kohler, J.; Simon, A.; Jepsen, O. Phys Rev B 1998, 57, 1510.

14. Blaha, P.; Schwarz, K.; Sorantin, P.; Trickey, S. B. Comput Phys Commun 1990, 59, 399.

15. Christensen, A.; Ruban, A. V.; Stoltze, P.; Jacobsen, K. W.; Skriver, H. L.; Norskov, J. K.; Besenbacher, F. Phys Rev B 1997, 56, 5822; Landa, A. I.; Ruban, A. V.; Abrikosov, I. A.; Wynblatt, P.; Skriver, H. L. In Structure and Evolution of Surfaces; Cammarata, R. C.; Chason, E. H.; Einstein, T. L.; Williams, S. D., Eds.; MRS Symp Proc 1997, 440, 467.

16. Ruban, A. V.; Skriver, H. L. Phys Rev B 1997, 55, 8801.

17. Connolly, J. W. D.; Williams, A. R. Phys Rev B 1983, 27, 5169.

18. Faulkner, J. S. Prog Mater Sci 1982, 27, 1.

19. Ruban, A. V.; Abrikosov, I. A.; Skriver, H. L. Phys Rev B 1995, 51, 12958.

20. Abrikosov, I. A.; Johansson, B. Phys Rev B 1998, 57, 14164. 